Simpósio de Geoestatística Aplicada em Ciências Agrárias
14 e 15 de maio de 2009
Botucatu-SP

\title{
GEOESTATÍSTICA NA ANÁLISE DA TEMPERATURA dO AR EM PETROLINA - PE
}

\section{Clovis Manoel Carvalho Ramos ${ }^{1}$, Luís Henrique Bassoi ${ }^{2}$, Alessandra Fagioli da Silva ${ }^{3}$, Anderson Antonio da Conceição Sartori ${ }^{4}$, Célia Regina Lopes Zimback ${ }^{5}$}

${ }^{1}$ Doutorando em Agronomia /Irrigação e Drenagem, Faculdade de Ciências Agronômicas, Universidade Estadual Paulista, - FCA-UNESP - clovisramos@fca.unesp.br.

${ }^{2}$ Pesquisador, Embrapa Semi-Árido - Inbassoi@cpatsa.embrapa.br.

${ }^{3}$ Mestranda em Agronomia/Energia na Agricultura, Faculdade de Ciências Agronômicas, Universidade Estadual Paulista, - FCA-UNESP - alefagioli@fca.unesp.br.

${ }^{4}$ Mestrando em Agronomia/Energia na Agricultura, Faculdade de Ciências Agronômicas, Universidade Estadual Paulista, - FCA-UNESP - sartori80@gmail.com.

${ }^{5}$ Professora, Faculdade de Ciência Agronômica, Universidade Estadual Paulista, - FCA-UNESP czimback@gmail.com.br.

Resumo - Este trabalho teve como objetivo estudar a dependência temporal na variação da temperatura do ar em Petrolina - PE, com diferentes horários de coleta de amostra, utilizando técnicas geoestatísticas e de estatística clássica. Dados de temperatura do ar de 2004, medidos a cada hora, foram distribuídos em três grupos: 0,8 e 16 horas; 2, 10 e 18 horas; e 4, 12, e 20 horas. Todos os dados de temperatura do ar apresentaram dependência temporal. A temperatura do ar horária estimada pelo modelo baseado nas medidas as 4,12 , e 20 horas foi que apresentou melhor estimativa da temperatura real medida em cada hora do dia. A observação da temperatura do ar em intervalos de 8 horas não se mostrou conveniente para a estimativa da temperatura do ar horária ao longo do dia.

Palavras-chave: analise temporal, krigagem.

GEOSTATISTICS IN THE ANALYSIS OF THE AIR TEMPERATURE IN PETROLINA, PE - BRAZIL

Abstract - This work aimed to study the dependence of the temporal variation of air temperature of Petrolina, Pernambuco State, Brazil, with different times of sample collection, using classical statistic and geostatistic. Air temperature data from 2004, measured every hour, were divided into three groups: 0, 8 and 16 hours, 2, 10 and 18 hours, and 4, 12, and 20 hours. All data of air temperature showed temporal dependence. The hourly air temperature estimated by the model based on the 4 measures, 12, and 20 hours presented the best estimation of the actual air temperature measured at every time of the day. The observation of air temperature at intervals of 8 hours was not appropriate to estimate the hourly air temperature throughout the day.

Key words: temporal analysis, kriging.

Introdução

O estudo da temperatura é de fundamental importância no setor agrícola, uma vez que interfere de forma relevante no rendimento das culturas. Caron et al. (2003) comentou que a eficiência das plantas em relação aos fluxos respiração e fotossíntese é influenciada pelas condições ambientais, principalmente, a radiação solar global incidente e a temperatura do ar. Desta forma os extremos de temperatura podem paralisar o desenvolvimento ou causar problemas fisiológicos das plantas.

O estudo dessa variável na região Nordeste tem importância no planejamento de atividades agrícolas devido ao estresse que as altas temperaturas provocam nas diversas culturas. Melhores previsões dos períodos em que podem ocorrer excesso de temperatura do ar permitem as tomadas de decisões mais confiáveis sobre vários aspectos das culturas. Para o estudo da temperatura do ar de uma região, além de uma série histórica, é importante a informação como ocorreu a variação de temperatura do ar ao longo do dia. Para tanto é necessário a utilização de equipamentos eletrônicos, o que em muitos casos inviabiliza a série histórica. A metodologia de coleta dos dados é de grande importância na melhor forma de estimar dados climáticos de determinada região.

O estudo da variabilidade espacial e temporal de uma variável de interesse pode ser feito através de técnica denominada geoestatística, que juntamente com a estatística clássica formam uma par de ferramentas de extraordinária importância agronômica, decorrente das inúmeras respostas dadas às mais 


\section{Simpósio de Geoestatística Aplicada em Ciências Agrárias}

14 e 15 de maio de 2009

Botucatu-SP

variadas questões existentes que, até então, eram ignoradas (CARVALHO et al., 2003). Este trabalho teve como objetivo estudar a distribuição temporal da variação da temperatura do ar em Petrolina- PE, com diferentes horários de coleta de amostra, visando a estimativa da temperatura do ar, utilizando técnicas geoestatísticas.

\section{Material e Métodos}

Os dados de temperatura do ar foram coletados em 2004 na Estação Agrometeorológica Automática de Bebedouro, localizada no Perímetro Irrigado de Bebedouro e pertencente a Embrapa Semi-Árido, PetrolinaPE. A estação está situada na latitude de $09^{\circ} 09^{\prime} \mathrm{S}$ e na longitude de $40^{\circ} 22^{\prime} \mathrm{W}$, com $365,5 \mathrm{~m}$ de altitude. $\mathrm{O}$ clima da região é do tipo BSwh, segundo a classificação de Köeppen, que corresponde a uma região climaticamente árida, com período chuvoso de janeiro a abril (TEIXEIRA et al., 2002). Os dados de temperatura média horária (T24) foram analisados, utilizando a técnica de geoestatística. A partir de dados de temperatura do ano de 2004 foram selecionados três conjuntos de dados pontuais com intervalos de oito horas entre cada amostragem para cada dia do ano. Os conjuntos dados diferiram entre si na hora da primeira observação: T0-8-16, com observações de temperatura as 0 , 8 e 16 horas; T2-10-18, com observações de temperatura as 2, 10 e 18 horas; e T4-12-20 com observações de temperatura as 4, 12, e 20 horas, respectivamente. Para os pontos não coletados, por falha do equipamento, foram mantidas as coordenadas do eixo horizontal e vertical sem valor de temperatura. Inicialmente, realizou-se uma análise descritiva dos dados para determinação dos parâmetros estatísticos: média; mediana; valor mínimo e máximo; desvio-padrão; coeficientes de variação, assimetria e curtose. A hipótese de normalidade foi verificada pelo teste de Kolmogorov-Smirnov. A análise geoestatística foi realizada com intuito de verificar a existência e quantificar o grau de dependência temporal entre as observações, com base na pressuposição de estacionariedade da hipótese intrínseca, a qual é estimada pela equação abaixo, segundo Vieira et al., (1983). As variâncias foram calculadas pela equação (1):

$$
\gamma *(h)=\left(\frac{1}{2 N(h)}\right) \sum_{i=1}^{N(h)}\left[Z\left(x_{i}\right)-Z\left(x_{1}+h\right)\right]^{2}
$$

em que: $\gamma^{*}(h)$ é a variância para um vetor $h($ dia); $Z(x)$ e $Z(x+h)$ são os pares de observações de temperatura, separados pelo vetor $h$ (dias); $N(h)$ é o número de pares de valores medidos $Z(x), Z(x+h)$, separados por um vetor $h$. A partir do ajuste de um modelo matemático são calculados os valores de $\gamma^{*}(h)$, e estimados os coeficientes efeito pepita $\left(\mathrm{C}_{0}\right)$, patamar $\left(\mathrm{C}_{0}+\mathrm{C}_{1}\right)$ e o alcance (a) do modelo teórico para o variograma. Os modelos foram ajustados pelo software $\mathrm{GS}^{+} 7.0$ (ROBERTSON, 2004). Em seguida, os variogramas foram escalonados pela variância dos dados, como descrito por Vieira (1983). Para verificar a dependencia temporal foi utilizado um indice de dependencia temporal (IDT) adaptado da metodologia proposta por Zimback (2001). Comprovada a dependência temporal realizou-se interpolação para estimar valores de temperatura com intervalo de uma hora, utilizando o método da krigagem ordinária. A fim de verificar a exatidão dos dados estimados foi confeccionado um mapa com a diferença de temperatura estimada, para cada conjunto de dados selecionado e os dados horários disponíveis utilizando o Sistema de Informação Geográfica Idrisi - Andes.

\section{Resultados e Discussão}

Os resultados da análise descritiva da temperatura estão apresentados na Tabela 1. Os dados de temperatura do ar, para o período observado no Perímetro Irrigado de Bebedouro, em Petrolina - PE, apresentaram assimetria positiva $\left(\mathrm{C}_{s}\right)$, com a média maior que a mediana. A exceção para o conjunto de dados T2-10-18, que apresentou assimétrico à direita.

Tabela 1. Estatística descritiva dos dados de temperatura do ar, em Petrolina - PE, em 2004

\begin{tabular}{lcccccccccc}
\hline Conjunto Dados & $\mathrm{n}$ & Média & Mediana & Mínimo & Máximo & $\mathrm{s}$ & $\mathrm{CV}$ & $\mathrm{C}_{\mathrm{s}}$ & $\mathrm{C}_{\mathrm{k}}$ & $\mathrm{d}$ \\
\hline T24 & 8616 & 26,09 & 25,54 & 14,9 & 38,75 & 4,03 & 15,45 & 0,33 & $-0,53$ & $0,06^{\text {ns }}$ \\
T0-8-16 & 1077 & 26,55 & 25,48 & 16,84 & 37,86 & 3,99 & 15,03 & 0,63 & $-0,52$ & 0,12 \\
T2-10-18 & 1077 & 26,01 & 26,38 & 16,46 & 34,29 & 3,25 & 12,50 & $-0,10$ & $-0,54$ & $0,05^{\text {ns }}$ \\
T4-12-20 & 1077 & 25,82 & 25,35 & 15,16 & 36,98 & 4,06 & 15,72 & 0,15 & $-0,80$ & $0,06^{\text {ns }}$ \\
\hline T4-24:
\end{tabular}

T24: observações da temperatura de hora em hora; T0-8-16: observações da temperatura as 0, 8 e 16 horas; T2-10-18: observações da temperatura as 2, 10 e 18 horas; T4-12-20: observações da temperatura as 4, 12 e 20 horas; $n$ : número de observações; s: desvio-padrão; CV: coeficiente de variação

(\%); Cs: Coeficiente de assimetria; Ck: coeficiente de curtose; d = teste de normalidade, ns: não significativo pelo teste de Kolmogorov-Smirnov. 


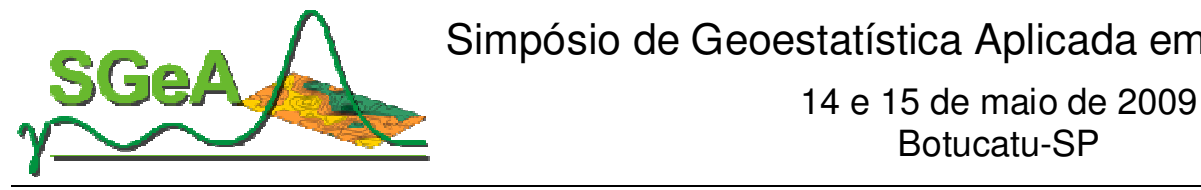

Todos os conjuntos de dados de temperatura do ar apresentaram distribuição de freqüência platicúrtica, com coeficiente de curtose menor que zero, mostrando uma tendência desses atributos apresentarem maior dispersão dos dados em torno da média. Com base no critério de Warrick e Nielsen (1980), o coeficiente de variação apresentou-se médio $(12 \%>\mathrm{CV}>60 \%)$ para todos os conjuntos de dados de temperatura do ar. Os resultados referentes ao teste de Kolmogorov-Smirnov indicaram distribuição normal para todos os conjuntos de dados, exceção para T0-8-16.

Os resultados da análise geoestatística (Tabela 2) mostram que os dados de temperatura do ar apresentaram dependência temporal, ajustando-se ao modelo esférico. Os pontos localizados num tempo menor ou igual ao alcance são mais similares e estão dependentes entre si, em relação tempo. Apenas o T4-12-20 apresentou valor de alcance próximo ao verificado nas medidas T24. Em relação ao alcance, observa-se que, os conjuntos de dados T0-8-16 e T2-10-18 foram os que apresentaram maior distorção em relação as medidas horárias (T24). Indicando que a amostragem de temperatura nos horários de 0, 8 e 16 horas e 2, 10 e 12 horas não representaram de forma exata a variação da temperatura ao longo do dia.

Tabela 2. Modelos e parâmetros dos variogramas escalonados da temperatura do ar

\begin{tabular}{llllllll}
\hline Conjunto Dados & Modelo & $\mathrm{Ao}$ & $\mathrm{C}_{0}$ & $\mathrm{C}_{0}+\mathrm{C}$ & $\mathrm{R}^{2}$ & $\mathrm{IDT}$ & $\mathrm{RCV}$ \\
\hline T24 & Esférico & 12,75 & 0,000625 & 0,957 & 94 & 99 & 0,95 \\
T0-8-16 & Esférico & 22,84 & 0,000625 & 1,08 & 77 & 99 & 0,88 \\
T2-10-18 & Esférico & 22,09 & 0,000946 & 0,883 & 82 & 99 & 0,84 \\
T4-12-20 & Esférico & 14,54 & 0,000607 & 0,927 & 68 & 99 & 0,88 \\
\hline
\end{tabular}

T24: observações da temperatura de hora em hora; T0-8-16: observações da temperatura as 0, 8 e 16 horas; T2-10-18: observações da temperatura as 2, 10 e 18 horas; T4-12-20: observações da temperatura as 4, 12 e 20 horas; Ao: alcance da dependência temporal; $\mathrm{C}_{0}$ : efeito pepita; $\mathrm{C}_{0}+\mathrm{C}$ : patamar; IDT: índice de dependência temporal e $\mathrm{R}^{2}$ : coeficiente de determinação múltipla do ajuste; RVC: coeficiente de determinação da validação cruzada.

Apesar dos valores do coeficiente de determinação da validação cruzada estarem muito próximos (Tabela 2), os modelos gerados pelo conjunto de dados T0-8-16 e T2-10-18 estimaram a temperatura do ar ao longo do dia com erro absoluto maior que a estimada pelo modelo gerado a partir do conjunto de dados T4-12-20 (Figura 1). Mesmo estimando a temperatura do ar ao longo do dia com maior exatidão, quando comparado aos demais, o modelo gerado a partir do conjunto de dados T4-12-20 apresenta uma estimativa com erro absoluto com até $4^{\circ} \mathrm{C}$, acima e abaixo da temperatura real observada. Enquanto a estimativa dos demais modelos chegou a $10^{\circ} \mathrm{C}$. O horário de medida da temperatura mostrou-se um importante variável na representação da variação deste fator climático ao longo do dia e conseqüentemente no ajuste do modelo que interpola a variação da temperatura.
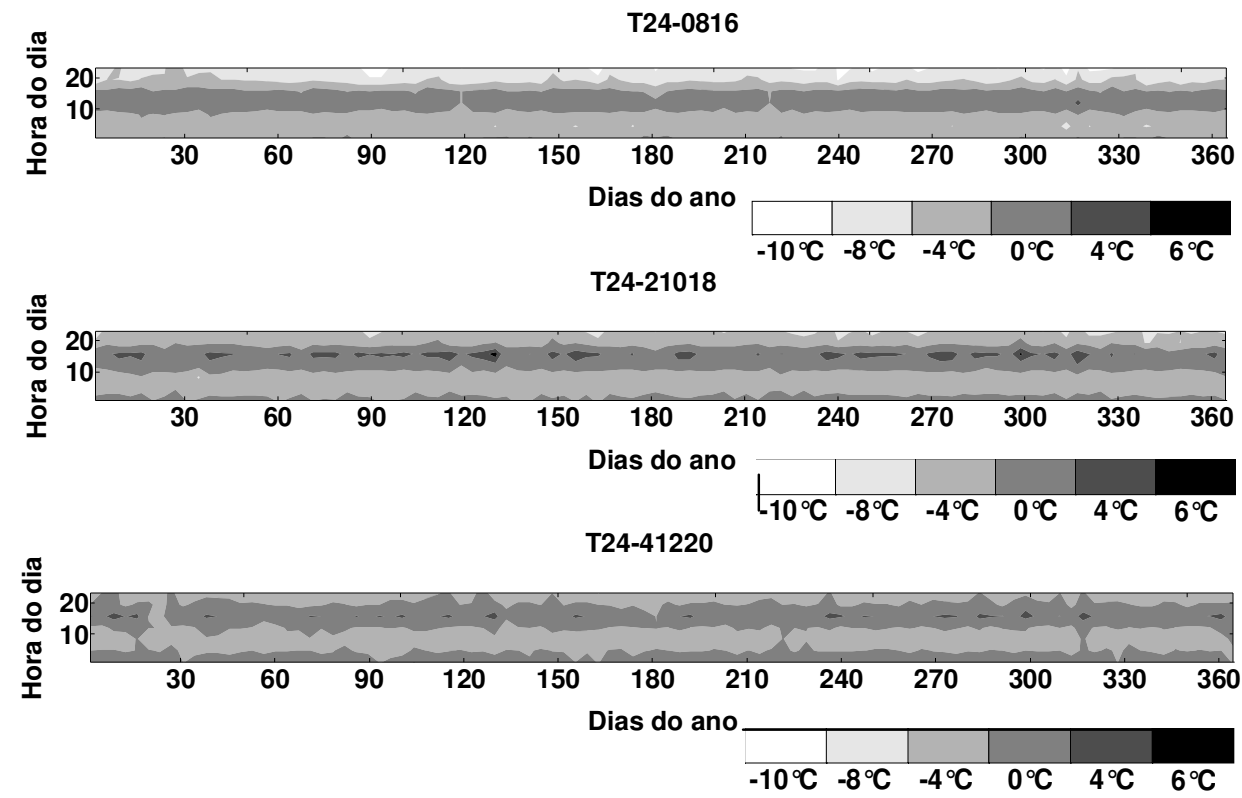

FIGURA 1. Mapa das diferenças da temperatura entre o modelo T24 e os modelos dos demais pontos de amostragem. 


\section{Conclusão}

Todos os dados de temperatura apresentaram dependência temporal. A temperatura do ar estimada pelo modelo T4-12-20 foi o que mais se aproximou da temperatura do ar real observada. O horário de registro da temperatura é fator importante na estimativa da temperatura ao longo do dia. É necessário testar outros intervalos a fim de reduzir o erro na estimativa. A geoestatística mostrou-se uma ferramenta útil na análise da variação da temperatura do ar.

\section{Agradecimentos}

A Embrapa Semi-Árido por ceder os dados.

\section{Referências}

CARON, B. O.; MEDEIROS, S. L. P.; MANFRON, P. A.; Schmidt, D.; POMMER, S. F.; Bianchi, C. Influência da temperatura do ar e radiação solar no acúmulo de fitomassa da alface. Revista Brasileira de Agrometeorologia, Santa Maria, v. 11, n. 2, p. 275-283, 2003. CARVALHO, M. P.; TAKEDA, E. Y.; FREDDI, O. S. Variabilidade espacial de atributos de um solo sob videira em Vitória Brasil (SP). R. Bras. Ci. Solo, Viçosa, vol. 27, n. 4, p. 695-703. 2003.

ROBERTSON, G. P. GS+: Geoestatistics for the environmental sciences - GS+ User's Guide. Plainwell, Gamma Desing Software, 2004. 152 p.

TEIXEIRA, A. H. de C.; BASSOI, L. H.; COSTA, W. P. L. B. da; SILVA, J. A. M.; SILVA, E. E. G. da. Consumo hídrico da bananeira no Vale do São Francisco estimado pelo método da razão de Bowen. Revista Brasileira de Agrometeorologia. Santa Maria, v. 10, n. 1, p. 45-50, 2002.

VIEIRA, S. R.; HATFIELD, T. L.; NIELSEN, D. R.; BIGGAR, J. W. Geostatistical theory and application to variability of some agronomical properties. Hilgardia, Oakland. v. 51, n. 3, p. 1-75, 1983.

WARRICK, A.W. e NIELSEN, D.R. Spatial variability of soil physical properties in the field. In: HILLEL, D. (Ed.). Application of soil physics. New York: Academic Press, 1980. 385 p.

ZIMBACK, C.R.L. Análise espacial de atributos químicos de solos para fins de mapeamento da fertilidade. 2001. 114 p. Tese de Livre-Docência (Livre-Docência em Levantamento do solo e fotopedologia), FCA/UNESP, 2001. 